\title{
The approach to the surgical management of cancer in four European countries
}

\author{
C.K. Connolly*, P.E.Y. Van Schil ${ }^{\#}$, R. Milroy", O. Braendli ${ }^{+}$, R.J. Prescott ${ }^{\S}$
}

The approach to the surgical management of cancer in four European countries. C.K. Connolly, P.E.Y. Van Schil, R. Milroy, O. Braendli, R.J. Prescott. (C)RS Journals Ltd 2003.

ABSTRACT: Outcome of lung cancer appears poorer in the UK than elsewhere in Europe. This may be due to a less aggressive approach in treatment. This study investigated whether clinicians' perceptions of their approach differed between European countries.

A questionnaire was circulated to cancer specialists in four countries (Belgium, Greece, Switzerland and the UK) asking about management. An aggression score was calculated using the proportion of standard cases that would proceed to operation at different ages and levels of pulmonary function.

The principle problems suggested by most of the 314 respondents were inoperability before symptoms (particularly in the UK) and confounding effects of comorbidity. Surgeons particularly blamed delay in referral. The aggression scores (Belgium 54\%, UK $49 \%$, Switzerland $47 \%$ and Greece $37 \%$ ) did not suggest the UK is an outlier, but the UK was more conservative in its approach to N2 disease and isolated cerebral metastasis. The aggression scores of surgeons were greater than those of the others $(51 \%$ versus $42 \%)$.

Lung cancer was felt to present late with potentially confounding symptoms, but delay in the clinical process was thought to be less important. Although the UK was more conservative with special cases, its approach to typical cases could account for differences in patient survival.

Eur Respir J 2003; 22: 838-844.
*Dept of Medicine, The Memorial Hospital, Darlington, UK, "\#ept of Thoracic and Vascular Surgery, University Hospital of Antwerp, Edegem, Belgium, Dept of Respiratory Medicine, Stobhill Hospital, Glasgow, ${ }^{+}$Zurcher Hohenklinik Wald, Faltigberg, Switzerland and ${ }^{\S}$ Medical Statistics Unit, University of Edinburgh, Edinburgh, UK.

Correspondence: C.K. Connolly, Aldbrough House, Aldbrough St John, Richmond, North Yorkshire, DL11 7TP, UK.

Fax: 441325374759

E-mail: ck-r.connolly@medix-uk.com

Keywords: Europe

lung cancer

operability

questionnaire

respectability

UK

Received: May 122003

Accepted: May 192003

This study was supported but not endorsed by a European Respiratory Society Task Force.
The published figures suggest that survival from cancer in general and lung cancer in particular is worse in the UK than most other European countries [1, 2]. Various reasons have been suggested for this. These include a less aggressive approach in the UK, possibly associated with underspending [3], later presentation and greater comorbidity, as compared with several other countries in Europe.

\section{Methods}

\section{General}

A postal questionnaire with one reminder was circularised to lung cancer specialists in Belgium, Greece, Switzerland and the UK. This had been piloted in the North-east of England.

\section{Recruitment}

Subjects were recruited as follows (the responsible members of the European Respiratory Society task force are shown in parentheses). 1) Belgium: two centres were contacted including all thoracic surgeons and physicians from the Belgium register of pulmonary physicians and the Belgian Association of Cardio-thoracic Surgery (P.E.Y. van Schil, V. Ninane); 2)
Greece: selected respiratory physicians and surgeons believed to have an interest in lung cancer (A. Rapti); 3) Switzerland: all pulmonary physicians, thoracic surgeons and radiotherapists believed to have an interest in lung cancer $(\mathrm{O}$. Braendli); 4) UK: all cancer specialists in Scotland (R. Milroy), Northeast of England, Yorkshire and East Anglia (C.K. Connolly) were identified from the British Thoracic Society list of respiratory physicians and by contact with the local cancer centres. Surgeons with a known interest in lung cancer throughout England and Wales were identified separately (D.A. Waller).

\section{Questionnaire}

The questionnaire consisted of three sections (see Appendix). It was emphasised to participants that they may have had little, if any, objective data but they were asked to state what they believe to be the case for the patients they actually saw from the area that they usually served. A) Perceptions concerning inoperability. In this section four possible answers were allowed: "never", "rarely", "sometimes" and "often". The questions covered: causes of inoperability: staging and comorbidity; the relationship between symptoms and onset of inoperability; and critical delay in the diagnostic and management process. B) The perceived proportion assessed as 
resectable and likely to proceed to surgery with radical intent in specific situations. The section was divided into three parts covering standard cases, special cases and the frequency of invasive investigations. In the first two sections an estimate of likelihood of operation was requested at the ages of 65,75 and $85 \mathrm{yrs}$, and when lung function were normal, 75 or $40 \%$ of predicted, giving nine potential answers for each example. The details of the cases were as follows. Standard cases: a coin lesion clear of the mediastinum and chest wall $2.5 \mathrm{~cm}$ in diameter; a segmental lesion suitable for right lower lobectomy with no lymph node involvement; a tumour in the right bronchial tree suitable for right pneumonectomy with no lymph node involvement. Special cases: a coin lesion involving rib laterally in the right mid chest; a tumour in the right upper lobe bronchus suitable for right upper lobectomy with ipsilateral N2 disease demonstrated preoperatively; an intrabronchial tumour suitable for right lobectomy with a cerebral metastasis which appears to be solitary after extensive investigation. The third subsection asked for the proportion of subjects with enlarged nodes on computed tomography (CT) scan proceeding to mediastinoscopy, mediastinotomy, or thoracoscopy. The possible answers were: "none"; "occasionally" ( $<5 \%)$; "less than half" (10-50\%); "more than half" (51-90\%); "virtually all" (>90\%). C) This section asked for details of the respondent's age, specialty and practice.

\section{Analysis}

Many of the results presented are simple tabulations. In section B the following scores representing approximate percentages of patients were used in certain analysis: none at all (0); occasional ( $<10 \%)(5)$; less than half (10-50\%) (30); more than half $(51-90 \%)(70)$; virtually all $(>90 \%)(95)$.

Further variables derived from the scores are introduced in the Results section. Comparisons of responses to individual questions across countries have been made using the KruskalWallis test, and between surgeons and nonsurgeons using the Wilcoxon Rank Sum test. Derived variables have been analysed using multiple regression. Rank correlation coefficients were used to test for the association between the number of patients seen and other variables.

\section{Results}

\section{General}

After exclusion of respondents not involved in cancer work or who were retired, and for duplicate requests, 314 satisfactory replies were received from $\sim 450$ participants, representing $\geqslant 65 \%$ of those circulated and eligible. Of those replying $4 \%$ were aged $<35,62 \% 35-50$, and $33 \%>50$ yrs.
Altogether, 51 were surgeons, but only one surgeon replied from Greece. There were few replies from the East Anglian region and preliminary analysis suggested that there was no difference between the replies from the North-east of England, Yorkshire and Scotland, and therefore UK replies were taken together. There was no natural division that gave balanced numbers of the nonsurgical specialties, so the results of the nonsurgical respondents were combined. This left four countries (Belgium $(n=46)$, Greece $(n=19)$, Switzerland $(n=103)$ and the UK $(n=146))$ and two groups of clinicians (surgeons $(n=51)$ and nonsurgeons $(n=263))$ to be considered.

\section{Section A: perceptions concerning inoperability}

In this section the original replies of "rarely" and "never" were combined to give three possibilities: "often", "sometimes" and "rarely or never". Table 1 gives the mean value for each response, assigning a score of 2 to "often", 1 to "sometimes" and 0 to "rarely or never". The implications of this are that a mean score of 1.3 or more indicates that the most frequent answer is likely to be "often", and if the score is $\leqslant 0.6$, likely to be "rarely or never". The p-values test for overall discrepancy in the distribution.

Staging and comorbidity. The mean score for "distant metastases" suggests that most respondents felt that these were "often" the cause of inoperability. Indeed the majority did reply this way. Most clinicians from all countries suggested that lymphatic spread at presentation was a common problem. The values shown in the tables suggest that pulmonary function "sometimes" restricted operability. This was true, except for Switzerland where, despite the mean score, the majority thought this was a rare problem. On the contrary, age was rarely felt to be relevant except in Greece, where the majority responded "sometimes". The UK score for "frailty" was three times higher than the mean of the other countries $(0.8$ versus 0.25 ) and only there was "sometimes" a frequent answer. Refusal of operation was universally considered a rare problem. With the exception "refused", the relatively small discrepancies between the replies of different countries were significant for all questions, suggesting that the replies within countries were very consistent. There was no discrepancy between the replies of surgeons and the rest in this question.

The relationship between the onset of symptoms and of inoperability. The replies suggested that early metastases and early local disease predating the first symptom were sometimes problems, particularly from the UK (table 2). Local spread after the first symptom, implying the development of inoperability after the first symptom, had a generally lower score than spread before symptoms, but in all countries it was accepted as "sometimes" being a problem. However, tumourrelated decline in general health resulting in inoperability was

Table 1. - The mean values of the answer scores to question 1 in section A: the frequency of the reasons for inoperability

\begin{tabular}{|c|c|c|c|c|c|c|c|c|c|}
\hline & Belgium & Greece & Switzerland & UK & p-value* & Surgeons & Others & p-value ${ }^{* *}$ & All \\
\hline Patients & 46 & 19 & 103 & 146 & & 51 & 263 & & 314 \\
\hline Distant metastases & 1.7 & 1.4 & 1.4 & 1.4 & 0.07 & 1.4 & 1.5 & 0.52 & 1.5 \\
\hline Local tumour, local nodes & 1.6 & 1.1 & 1.3 & 1.7 & 0.0001 & 1.4 & 1.5 & 0.05 & 1.5 \\
\hline Pulmonary function & 0.8 & 0.4 & 0.6 & 1.1 & 0.0001 & 0.8 & 0.9 & 0.38 & 0.9 \\
\hline Tumour debility & 0.2 & 0.2 & 0.3 & 0.6 & 0.0001 & 0.3 & 0.4 & 0.34 & 0.4 \\
\hline General frailty & 0.2 & 0.1 & 0.3 & 0.8 & 0.0001 & 0.5 & 0.5 & 0.97 & 0.5 \\
\hline Too old & 0.2 & 0.6 & 0.2 & 0.3 & 0.004 & 0.2 & 0.3 & 0.34 & 0.3 \\
\hline Refused & 0.0 & 0.1 & 0.1 & 0.1 & 0.61 & 0.1 & 0.1 & 0.21 & 0.1 \\
\hline
\end{tabular}

Data are presented as $\mathrm{n}$ unless otherwise stated. *: difference between countries; **: difference between surgeons versus the rest. 
Table 2. - The mean values of the answer scores to question 2 section A: when and why patients who are inoperable because of the cancer itself become inoperable

\begin{tabular}{|c|c|c|c|c|c|c|c|c|c|}
\hline & Belgium & Greece & Switzerland & UK & p-value* & Surgeons & Others & p-value** & All \\
\hline Patients & 46 & 19 & 103 & 146 & & 51 & 263 & & 314 \\
\hline Distant metastasis & 1.2 & 1.3 & 1.1 & 1.2 & 0.86 & 1.2 & 1.2 & 0.57 & 1.2 \\
\hline Local disease, no metastases & 1.1 & 1.0 & 1.0 & 1.4 & 0.002 & 1.1 & 1.2 & 0.67 & 1.2 \\
\hline Post symptom local spread & 0.8 & 1.0 & 0.7 & 0.9 & 0.05 & 0.7 & 0.8 & 0.34 & 1.2 \\
\hline Post symptom debility & 0.2 & 0.4 & 0.2 & 0.3 & 0.03 & 0.2 & 0.2 & 0.82 & 0.2 \\
\hline
\end{tabular}

Data are presented as $\mathrm{n}$ unless otherwise stated. *: difference between countries; **: difference between surgeons versus the rest.

not regarded as an appreciable problem anywhere. The small discrepancies between the countries were again statistically significant, with no difference between surgeons and the others.

The timing of the critical delay in patients who become inoperable between the first symptom and completion of assessment. Delay before consulting after the first symptom was felt sometimes to be a problem with a highly significant difference between surgeons (most frequent response "often"), and the others (table 3). All agreed that confusion by chronic disease was at least as, if not more important. Here there was no difference between the physicians and the surgeons. The perceptions of the importance of referral delay from primary care varied between "rare" and "sometimes" with surgeons feeling it much more important than others. Although little weight was put on delay during assessment in any country, it was perceived to be a problem in the UK three times more often than elsewhere (score 0.3 versus $0.1, \mathrm{p}=0.0001$ )

\section{Section B: the likelihood of operation in specific cases}

The respondent's replies are summarised in tables 4-6, which give the proportion of clinicians likely to recommend operation on half or more of subjects.

Typical cases. There was a consistent reduction in the likelihood of operation as the severity of the lesion and the age of the patient increased and the pulmonary function decreased (table 4). Although the scales are not strictly comparable, pulmonary function and age appeared to be given equal weight. Surgeons were more likely to recommend operation than the rest in all but the oldest patients with the poorest function.

Special cases. There was a similar trend with age and pulmonary function in the special cases (table 5). However, surgeons felt patients were more likely to have an operation on

Table 3. - The mean values of the answer scores to question 3 in section A: the timing of the critical delay in patients who become inoperable between the first symptom and completion of assessment

\begin{tabular}{lccccccccccc}
\hline & Belgium & Greece & Switzerland & UK & p-value* & Surgeons & Others & p-value** & All & All \\
\hline Patients & 46 & 18 & 103 & 146 & & 51 & 263 & & 314 \\
Confused by chronic disease & 1.2 & 0.9 & 1.1 & 1.3 & 0.05 & 1.3 & 1.2 & 0.19 & 1.3 & 1.2 \\
Delay before consulting & 1.0 & 0.4 & 1.0 & 1.1 & 0.0007 & 1.4 & 1.0 & 0.0007 & 1.4 & 1.0 \\
Referral delay & 0.6 & 0.5 & 0.4 & 0.6 & 0.06 & 0.9 & 0.5 & 0.0003 & 0.9 & 0.5 \\
Waiting time & 0.4 & 0.2 & 0.3 & 0.2 & 0.22 & 0.4 & 0.2 & 0.008 & 0.4 & 0.2 \\
Delay during assessment & 0.1 & 0.1 & 0.0 & 0.3 & 0.0001 & 0.2 & 0.1 & 0.10 & 0.2 & Others \\
\hline
\end{tabular}

Data are presented as $\mathrm{n}$ unless otherwise stated. *: difference between countries; ${ }^{* *}$ : difference between surgeons versus the rest.

Table 4. - The percentage of respondents expecting operation in $50 \%$ of patients

Lung function

$100 \%$

$70 \%$

$40 \%$

Surgeons Other disciplines All subjects Surgeons Other disciplines All subjects Surgeons Other disciplines All subjects

\begin{tabular}{|c|c|c|c|c|c|c|c|c|c|}
\hline \multicolumn{10}{|c|}{ Coin Lesion } \\
\hline \multicolumn{10}{|c|}{ Age yrs } \\
\hline 65 & 100 & 94 & 95 & 98 & 92 & 93 & 56 & 39 & 41 \\
\hline 75 & 96 & 87 & 89 & 90 & 74 & 77 & 27 & 14 & 17 \\
\hline 85 & 60 & 33 & 37 & 41 & 22 & 25 & 4 & 4 & 4 \\
\hline \multicolumn{10}{|c|}{ Lobectomy } \\
\hline \multicolumn{10}{|c|}{ Age yrs } \\
\hline 65 & 100 & 93 & 94 & 98 & 87 & 90 & 47 & 32 & 35 \\
\hline 75 & 96 & 81 & 94 & 86 & 68 & 70 & 14 & 11 & 11 \\
\hline 85 & 56 & 29 & 33 & 37 & 19 & 22 & 2 & 3 & 3 \\
\hline \multicolumn{10}{|c|}{ Pneumonectomy } \\
\hline \multicolumn{10}{|c|}{ Age yrs } \\
\hline 65 & 100 & 88 & 90 & 92 & 73 & 76 & 25 & 9 & 12 \\
\hline 75 & 84 & 60 & 64 & 62 & 34 & 39 & 10 & 3 & 5 \\
\hline 85 & 28 & 13 & 15 & 16 & 8 & 8 & 0 & 3 & 2 \\
\hline
\end{tabular}


Table 5. - The percentage of respondents expecting operation in $50 \%$ of patients

\begin{tabular}{|c|c|c|c|c|c|c|c|c|c|}
\hline & \multicolumn{9}{|c|}{ Lung function } \\
\hline & \multicolumn{3}{|c|}{$100 \%$} & \multicolumn{3}{|c|}{$70 \%$} & \multicolumn{3}{|c|}{$40 \%$} \\
\hline & Surgeons & $\begin{array}{c}\text { Other } \\
\text { disciplines }\end{array}$ & $\begin{array}{c}\text { All } \\
\text { subjects }\end{array}$ & Surgeons & $\begin{array}{c}\text { Other } \\
\text { disciplines }\end{array}$ & $\begin{array}{c}\text { All } \\
\text { subjects }\end{array}$ & Surgeons & $\begin{array}{c}\text { Other } \\
\text { disciplines }\end{array}$ & $\begin{array}{c}\text { All } \\
\text { subjects }\end{array}$ \\
\hline \multicolumn{10}{|c|}{ Chest wall lesion } \\
\hline \multicolumn{10}{|c|}{ Age yrs } \\
\hline 65 & 100 & 71 & 79 & 98 & 66 & 73 & 33 & 23 & 24 \\
\hline 75 & 86 & 48 & 58 & 70 & 38 & 43 & 16 & 8 & 9 \\
\hline 85 & 34 & 12 & 16 & 16 & 8 & 9 & 2 & 5 & 5 \\
\hline \multicolumn{10}{|c|}{ N2 lesion } \\
\hline \multicolumn{10}{|c|}{ Age yrs } \\
\hline 65 & 22 & 63 & 50 & 18 & 43 & 42 & 0 & 9 & 8 \\
\hline 75 & 11 & 36 & 32 & 4 & 26 & 23 & 0 & 3 & 3 \\
\hline 85 & 0 & 6 & 5 & 0 & 4 & 4 & 0 & 1 & 1 \\
\hline \multicolumn{10}{|c|}{ Cerebral metastasis } \\
\hline Age y & & & & & & & & & \\
\hline 65 & 32 & 23 & 25 & 27 & 16 & 18 & 8 & 4 & 4 \\
\hline 75 & 17 & 10 & 11 & 15 & 7 & 8 & 2 & 1 & 1 \\
\hline 85 & 2 & 3 & 3 & 2 & 2 & 2 & 0 & 1 & 1 \\
\hline
\end{tabular}

an isolated lesion invading the chest wall than nonsurgeons. The opposite applied to $\mathrm{N} 2$ disease.

Aggression score. In order to compare the approach in different countries, and of surgeons with the rest, the approach to surgery was quantified over the range of the typical scenarios described. The mean score for all 27 potential answers to the three typical cases was calculated for each individual and termed the "aggression score". There was little difference between the scores of the surgeons in different countries (mean score $54 \%$ ), but the score of the nonsurgeons varied from $32 \%$ for Greece through $42 \%$ and $44 \%$ for Switzerland and the $\mathrm{UK}$, respectively, to $50 \%$ for Belgium. On multiple regression analysis the difference between surgeons and nonsurgeons after adjustment for country was highly significant at $52 \%$ versus $42 \%(\mathrm{p}<0.001)$. The adjusted scores for country after correction for surgeon and nonsurgeon were Belgium 54\%, UK $49 \%$, Switzerland $47 \%$ and Greece $37 \%$. These scores for Belgium are significantly more aggressive than the others ( $p>0.006$ ) except the UK, and for Greece significantly less aggressive than any other country $(\mathrm{p}<0.01)$.

Intervention score. A similar "intervention score" was designed to assess the likelihood of operation in special circumstances. This was calculated from the replies for the special cases. This score is not valid for comparison between surgeons and nonsurgeons because of the different pattern of replies to the questions on chest wall and N2 disease. Nevertheless, as this score reflects the global approach to these special cases where surgical intervention might be considered to be controversial, it may still be appropriate for comparison between countries. On multivariate analysis the difference was highly significant after correction for surgeons and nonsurgeons. The intervention scores were as follows: Switzerland $35 \%$, Belgium 26\%, Greece $22 \%$ and UK 19\%. The score is significantly greater for Switzerland than for any other country $(p=0.002)$ and the score of the UK is significantly less than that for Belgium $(\mathrm{p}=0.008)$. The replies from the $\mathrm{UK}$ for the coin lesion invading the chest wall were virtually identical to the average, so the low UK score is entirely due to reluctance to recommend surgery in $\mathrm{N} 2$ disease or in the presence of an isolated cerebral metastasis.

Implications for operation rates. The questionnaire was designed to test the approach of clinicians to management, and table 4 summarises the responses in terms of the percentage of respondents anticipating each level of surgical activity. From the patients' perspective, this might give a false impression of the proportion of individuals actually likely to have an operation. To test this, the authors have reanalysed question $1 \mathrm{~b}$ : lobectomy. Table 6 shows the proportion of patients assessed as resectable by lobectomy, likely to proceed to surgery. This made only a small difference, but suggested that the method of presentation of the results in tables 4 and 5 slightly overestimates the proportion of young subjects with good function perceived as actually proceeding to surgery, whilst slightly underestimating the proportion of the elderly with poor function in whom operation is undertaken.

Table 6. - The percentage of subjects who would have surgery

\begin{tabular}{|c|c|c|c|c|c|c|c|c|c|}
\hline & \multicolumn{9}{|c|}{ Lung function } \\
\hline & \multicolumn{3}{|c|}{$100 \%$} & \multicolumn{3}{|c|}{$70 \%$} & \multicolumn{3}{|c|}{$40 \%$} \\
\hline & Surgeons & Other disciplines & All subjects & Surgeons & Other disciplines & All subjects & Surgeons & Other disciplines & All subjects \\
\hline \multicolumn{10}{|c|}{ Lobectomy } \\
\hline \multicolumn{10}{|c|}{ Age yrs } \\
\hline 65 & 94 & 86 & 88 & 92 & 82 & 84 & 46 & 34 & 36 \\
\hline 75 & 90 & 75 & 77 & 81 & 62 & 65 & 23 & 19 & 19 \\
\hline 85 & 57 & 32 & 36 & 37 & 24 & 26 & 6 & 6 & 6 \\
\hline
\end{tabular}


Mediastinoscopy, mediastinostomy and thoracoscopy (mediastinal nodes present). Altogether, 34 (of 51) surgeons, and 79 (of 263) others, would recommend one of these procedures before operation in virtually all cases, and 42 and 155 , respectively in more than half of the cases. There was little difference in the distribution between countries.

\section{Section C: age, specialty and practice demography}

The authors looked at the distribution of the answers to question 1 and the aggression score in the light of the above three factors. Because of the difference in the replies of physicians and surgeons the components of the intervention score were considered separately. There was no relationship between the replies to the questions in the first two sections and most of the details of the age and practice in individuals. The one relevant factor appeared to be the caseload in the institutions where individuals were working. The aggression score consistently increased from 42 , when $<25$ people were managed annually, to 50 where $>150$ cases were discussed $(\mathrm{p}=0.003)$. Furthermore, the greater the number of patients seen, the more likely nonsurgeons were to agree with the surgeons about the two relevant special cases. With increasing workload ( $\leqslant 25,26-50,51-150$ and $>150$ cases seen annually) the mean scores for chest wall involvement were 27, 33, 36 and 46 , respectively $(\mathrm{p}<0.001)$, while for $\mathrm{N} 2$ node operability the mean scores consistently decreased $(24,23,19$ and 16 , respectively; $\mathrm{p}<0.0001)$.

\section{Discussion}

The response rate to the questionnaire was relatively high $(65 \%)$ and therefore probably gives a fair reflection of the views of lung cancer clinicians in the countries concerned. The answers give the respondents' opinions and are not validated, but nevertheless there is a suggestion that some of the perceptions about differences between the countries are wrong. In particular the perception of a generally less aggressive approach in the UK is not supported by these findings.

In the first section it was clear that the respondents felt that many patients were inoperable before they developed their first symptom. The only way to improve outcome in these patients is to set up a satisfactory screening service. There are early indications that spiral CT may be of value [4]. This seems to be most useful in detecting peripheral nodules whose prognosis is likely to be good. The potential for improvement in long-term outcome may therefore be small. The intervention may simply bring forward the time of diagnosis in patients who would remain operable for a long time. Experience from subsequent interval examinations, where far fewer tumours were detected [5], suggests that this might be the case. After peripheral nodule, the next most favourable presentation of lung cancer is segmental intrabronchial tumour suitable for lobectomy [6]. For cancer and noncancer reasons, the prognosis is much more favourable when lobectomy is still possible at the time of operation [7]. These intrabronchial lesions may be less easy to pick up on CT scanning. The patients often present with recurrent infection, which may be similar to their chronic symptoms, and so the cancer might be missed and become inoperable during the symptomatic period. This interpretation agrees with the view of the respondents who feel that inoperability after the first symptom because of the confounding effects of other symptoms or delay in obtaining advice may be a problem. In the absence of haemoptysis it is easy to understand how the symptom may be interpreted as being very similar to those of the preceding years. There is a clear need for research into the pattern of development of symptoms in patients at risk of lung cancer.

Few of the respondents, even in the UK, felt that the process delay was now important in determining outcome. If this is the case, then the disadvantages of attempting to shorten the investigation period further becomes important. First, undue haste in the investigation process in an attempt to reach unnecessary targets may result in inappropriate staging. Secondly, fluctuations in presentation, holidays and staff sickness mean that short delays are inevitable. When these occur undue emphasis on rapid process might cause additional worry to patients and relatives. Thirdly, where clinicians are involved in other respiratory work the services might be transferred from noncancer patients unnecessarily. There is a clear need for further research to establish whether there is any relationship between outcome and the interval between presentation and definitive procedure, and if so, whether there is a group of patients where "fast tracking" is justified for reasons other than severe symptoms.

Chronological age [8], in contrast to performance status, is not important in determining outcome. On the contrary, pulmonary function is important and there are clear recommendations to ensure adequate pulmonary function before surgery [9]. There was an apparent discrepancy between the replies with regard to the weight given to age and to pulmonary function in the first section, and the actual replies given for specific clinical examples in the second section. In general, pulmonary function was felt "sometimes" to be the limiting factor, but age "rarely" so. Although the scales are not strictly comparable, equal emphasis appears to have been put upon age and pulmonary function when considering operability. However, if frailty amounts to biological age, and the scores for age and frailty in table 1 are combined, the balance is at least partially redressed. This would be true for the UK in particular where the "frailty" score was highest and may reflect greater comorbidity in that country than elsewhere. Perhaps the participants had also been unconsciously "politically correct" when replying to the first section in claiming not to take age into consideration. This emphasises the need to treat the perceptions recorded in that section with caution.

There is qualified support for patients to be seen by experienced clinicians. In the one example where the surgeons were less aggressive than the nonsurgeons (operating on effectively inoperable N2 disease) the decisions of those who saw more patients agreed with the surgeons, despite the fact they were in general more aggressive.

The results do not support the view that the approach to the surgery of standard cases is different between the three North European countries, despite the difference in availability of resources. It was suggested particularly by the surgeons that operations are done more readily than the literature suggests should be the case e.g. an absolute contraindication at pulmonary function of $<50 \%$ predicted [9]. The perceived differences in the management of the standard cases were not consistent with the observed differences in survival (Switzerland relatively good, Belgium average and UK poor [1,2]), suggesting that a less aggressive approach to standard cases in the UK is not the reason for the differences in survival. Furthermore, although the number of clinicians prepared to operate on chest wall disease, the special case where the outlook is good [10], was disappointing, the UK was no worse than the other countries in this respect. However, it is clear that the clinicians in the UK are much less likely to intervene in the other two special cases, N2 disease where surgery is rarely if ever justified [11] and in the presence of an isolated cerebral secondary, where surgery sometimes 
may be appropriate [12]. Even if operations are occasionally successful, radical surgery in N2 disease is unlikely to have any major impact on outcome, and isolated brain secondaries are rare. These cases may require a disproportionate use of resources, so perhaps in these instances the more parsimonious approach of the UK is justified, even if it is in part determined by the resources available. Although, other clinicians may be less inclined than surgeons to recommend invasive staging procedures pre-operatively, despite their known value [13, 14], there were no differences between the countries. Overall, the replies gave little credence to the view that survival in lung cancer is shorter in the UK than in the other countries because of a less aggressive approach to the management of the disease, and suggest one factor may be greater frailty or comorbity in the UK lung cancer population.

\section{Appendix: European task force questionnaire surgical resection rates for bronchial carcinoma}

\section{A. Inoperability: reasons and timing}

In this section, we are asking you what you believe to be the case in the light of your experience in managing lung cancer. We accept that you may have little if any objective data. We are asking you about the patients that you and your group actually see from the area that you usually serve.

Key for answers: never $=0$, rarely $=1$, sometimes $=2$, often $=3$

A.1 Please give your impression of the frequency of reasons for inoperability as listed below:

Inoperable, distant metastases

Inoperable, local tumour/local nodes

Otherwise operable, pulmonary function inadequate

Otherwise operable, too ill, directly related to tumour

Otherwise operable, too frail generally

Otherwise operable, too old

Operable, refused operation

A.2 Of the patients who are inoperable because of the cancer itself, please say when and why you believe they became inoperable:

Inoperable before the first symptom because of distant metastases

Inoperable before the first symptoms because of local disease without distant metastases

Became inoperable after the first symptom because of local disease without distant metastases

Became inoperable after the first symptom only because of tumour-related debility

A.3 Of those patients who became inoperable between the first symptom and completion of assessment, please indicate where you feel the critical delay occurs:

Patient's failure to recognise new symptoms because of chronic respiratory symptoms

Interval between recognition of first symptom and first consultation in primary care

Interval between first consultation in primary care and decision to refer

Interval between referral from primary care/self referral and specialist assessment.

Interval between specialist assessment and definitive treatment

\section{B. Criteria for operability}

We are asking you about what happens to the patients that your group sees, whatever your contribution to the final management plan. The questions below allow for chronological age and pulmonary function. In giving your answer please consider all patients with whom your group has contact, including those who were inoperable because of other respiratory and non-respiratory co-morbidities. We have deliberately given a range to allow for differences in general health, etc.

B.1 In your practice, what proportion of patients satisfying the criteria below at the time of final assessment would proceed to surgery with a view to complete excision? It should be assumed that your usual investigations have not revealed evidence of distance growth.

Key for answers:

$\begin{array}{ll}\text { none at all } & =0 \\ \text { occasional }(<10 \%) & =1 \\ \text { less than half }(10-50 \%) & =2 \\ \text { more than half }(51-90 \%) & =3 \\ \text { virtually all }(>90 \%) & =4\end{array}$

(a) A coin lesion clear of the mediastinum and chest wall $2.5 \mathrm{~cm}$ in diameter

$\begin{array}{cccc} & \text { function normal } & \text { function } 75 \% & \text { function } 40 \% \\ \text { Age } 65 & {[]} & {[]} & {[]} \\ \text { Age } 75 & {[]} & {[]} & {[]} \\ \text { Age } 85 & {[]} & {[]} & {[]}\end{array}$

(b) A segmental lesion suitable for right lower lobectomy with no lymph node involvement

function normal function $75 \%$ function $40 \%$

$\begin{array}{cccc}\text { Age } 65 & {[]} & {[]} & {[]} \\ \text { Age } 75 & {[]} & {[]} & {[]} \\ \text { Age } 85 & {[\text { ] }} & {[]} & {[]}\end{array}$

(c) A tumour obstructing the right bronchial tree suitable for right pneumonectomy with no lymph gland involvement

$\begin{array}{cccr} & \text { function normal } & \text { function } 75 \% & \text { functio } \\ \text { Age } 65 & {[]} & {[]} & {[]} \\ \text { Age } 75 & {[]} & {[]} & {[]} \\ \text { Age } 85 & {[]} & {[]} & {[]}\end{array}$

B.2 In what proportion of patients might radical surgery be attempted in the following special situations?

Key for answers:

$\begin{array}{ll}\text { none at all } & =0 \\ \text { occasional }(<10 \%) & =1 \\ \text { less than half }(10-50 \%) & =2 \\ \text { more than half }(51-90 \%) & =3 \\ \text { virtually all }(>90 \%) & =4\end{array}$

(a) A coin lesion invading rib laterally in the mid right chest function normal function $75 \%$ function $40 \%$

$\begin{array}{llll}\text { Age } 65 & {[]} & {[]} & {[]} \\ \text { Age } 75 & {[]} & {[]} & {[]} \\ \text { Age } 85 & {[]} & {[]} & {[]}\end{array}$

(b) A tumour obstructing the right upper lobe bronchus suitable for right upper lobectomy with ipsilateral N2 disease demonstrated pre-operatively

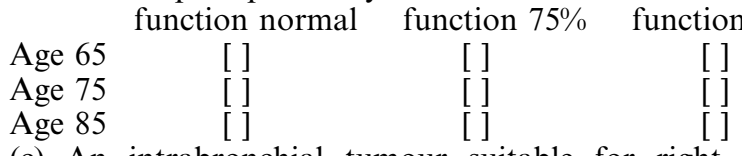

(c) An intrabronchial tumour suitable for right lower lobectomy with cerebral metastases which appears to be solitary after extensive investigation

$\begin{array}{cccr} & \text { function normal } & \text { function } 75 \% & \text { functio } \\ \text { Age } 65 & {[]} & {[]} & {[]} \\ \text { Age } 75 & {[]} & {[]} & {[]} \\ \text { Age } 85 & {[]} & {[]} & {[]}\end{array}$

B.3 What proportion of patients seen by you with bronchoscopably operable tumour and lymph node enlargement on CT scan without evidence of extra-thoracic metastases undergo mediastinoscopy/mediastinotomy/thoracoscopy? 
Key for answers:

none at all occasional $(<10 \%)$

$=0$ less than half $(10-50 \%)$ more than half $(51-90 \%)$ virtually all $(>90 \%)$

$=1$

$=2$

$=3$

$=4$

C. Please give us the following details about yourself and the place you work by ticking the appropriate boxes:

\begin{tabular}{|c|c|c|}
\hline C.1 Age $\underset{35-5}{<}$ & years [] & $\begin{array}{ll}51-65 & {[]} \\
>65 \text { years } & {[]}\end{array}$ \\
\hline C.2 Specialty & Physician - & $\begin{array}{l}\text { Respiratory } \\
\text { Other }\end{array}$ \\
\hline & Oncologist - & $\begin{array}{l}\text { Chemotherapy only } \\
\text { Other }\end{array}$ \\
\hline & Surgeon - & $\begin{array}{l}\text { Pure thoracic } \\
\text { Other } \\
\text { Other }\end{array}$ \\
\hline
\end{tabular}

C.3 Selection of patients: please tick the description which is closest to the sample that you report

Unselected self referrals

Unselected referrals from primary care

Overview of patients referred to your institution from other specialists

Restricted to your specialty e.g. surgery, radiotherapy [ ]

Participation in multidisciplinary meetings without direct clinical responsibility

C.4 Approximately how many lung cancer patients do you see and/or discuss at multidisciplinary meetings?

$\begin{array}{llll}<25 & {[]} & 25-75 & \text { [ ] } \\ 76-150 & {[]} & >150 & \text { [ ] }\end{array}$

C. 5 How far is the surgical unit from your centre?

At your centre [ ] $<10$ miles $/<15 \mathrm{~km}$ $10-30$ miles $/ 15-50 \mathrm{~km} \quad[$ ] $31-60$ miles $/ 50-100 \mathrm{~km}$ [] $>60 \mathrm{miles} />100 \mathrm{~km} \quad[$ ]

C.6 Economic status of patients in your practice
a) Deprived
[ ] b) Average
c) Affluent
[ ]

C.7 Geographical source of your patients
a) Mainly rural
[]
b) Industrial city
c) Non-industrial city []
d) Mixed

Thank you very much indeed for filling in this form - please write any comments on the back of this page and return it in the freepost envelope.

\footnotetext{
Acknowledgements. The authors would like to acknowledge the help of C. Paul with the analysis, the European Respiratory Society for a generous
}

grant, and the participants whose replies made the study possible.

\section{References}

1. Berrino F, Sant M, Verceccia A, Caporaccia R, Hakulinen T, Esteve J. Survival of cancer patients in Europe. The EUROCARE study. IARC Scientific Publications No. 132, Lyon, International Agency for Research on Cancer, 1995.

2. Janssen-Heijnen MLG, Gatta G, Forman D, Capocaccia R, Coebergh JWW, and the EUROCARE working group. Variation in survival of patients with lung cancer in Europe, 1985-1989. Eur J Cancer 1998; 34: 2191-2196.

3. The Office of Health Economics Compendium of Health Statistics. 13th edn. London, HM Stationary Office, 2001.

4. Henschke CI, McCauley DI, Yankelevitz DF, et al. Early lung cancer action project: overall design and findings from baseline screening. Lancet 1999; 354: 99-105.

5. Henschke CI, Naidich DP, Yankelevitz DF, et al. Early lung cancer action project: initial findings on repeat screenings. Cancer 2001; 92: 153-159.

6. Scagliotti GV. Symptoms and signs of stagings of lung cancer. Eur Respir Mon 2001; 6(17): 86-119.

7. Van Rens MTHM, Brutel de la Riviere A, Elbers HRJ, van den Bosch JMM. Prognostic assessment of 2,361 patients who underwent pulmonary resection for non-small cell lung cancer, stage 1, 11 and 111A. Chest 2000; 117: 374 379 .

8. Skuladottir H, Olsen JH. Epidemiology of lung cancer. Eur Respir Mon 2001; 6(17): 1-12.

9. Bolliger CT, Perruchoud AP. Functional evaluation of the lung resection candidate. Eur Respir J 1998; 11: 198-212.

10. Goldstraw P, Mannam GC, Kaplan DK, Michail P. Surgical management of non-small cell lung cancer with ipsilateral mediastinal node metastases (N2 disease). J Thoracic Cardiovasc Surg 1994; 107: 19-28.

11. Downey RJ, Martini N, Rusch VW, et al. Extent of chest wall invasion and survival in patients with lung cancer. Ann Thorac Surg 1999; 68: 188-193.

12. Burt M, Wronski M, Arbit E, et al. Resection of brain metastases from non-small cell lung carcinoma: results of therapy. J Thorac Cardiovasc Surg 1992; 103: 399-410.

13. Van Schil P, Van den Brande F. The current role of invasive staging in lung cancer. Monaldi Arch Chest Dis 1997; 52: 237-241.

14. Keller SM, Adak S, Wagner $\mathrm{H}$, et al. Mediastinal lymph node dissection improves survival in patients with stages II and IIIA non-small cell lung cancer. Ann Thorac Surg 2000; 70: $358-366$. 